Die folgenden Beiträge der Hausärzte Franz Marty und Bernhard Sorg setzen sich kritisch mit dem Weiterbildungsprogramm zum Facharzt für Allgemeine Innere Medizin auseinander, das aus ihrer Sicht in Bezug auf die Sicherstellung des hausärztlichen Nachwuchses und die hausärztlichen Kompetenzen Mängel aufweist. Im Sinne einer breiteren Diskussion der angesprochenen Fragen hat die Redaktion die Schweizerische Gesellschaft für Allgemeine Innere Medizin (SGAIM) als zuständige Fachgesellschaft und weitere Experten zu Stellungnahmen eingeladen. Sie finden die Beiträge als Themenschwerpunkt auf den folgenden Seiten.

Die Redaktion

\title{
Ein Feedback aus der Praxis
}

\section{Franz Marty}

Dr. med., Mitglied FMH

\section{Einleitung}

In der ambulanten ärztlichen Versorgung der Schweiz blieb während der letzten 15 Jahre kaum ein Stein auf dem andern! Der strukturelle Wandel in der Praxislandschaft [1] und die akademische Verankerung eröffneten den jungen Hausärztinnen und -ärzten neue berufliche Laufbahnen und akademische Karrieren. Hausarztmedizin ist heute die Disziplin mit den vielfältigsten beruflichen Möglichkeiten, bei gleichzeitig sehr flexiblen Arbeitsmodellen [2]!

Trotz dieser attraktiven Ausgangslage verschärfte sich die Nachfolgeproblematik in den letzten Jahren stark. Warum wählen nicht mehr Ärzte die «Laufbahn HA»? Spurt das neue Weiterbildungsprogramm (WBP) «Allgemeine Innere Medizin» (AIM) zu stark in Richtung "stationärer Sektor»? Oder ist die hausärztliche Weiterbildung zuwenig attraktiv?

\section{Weiterbildung in Hausarztmedizin}

Die meisten Länder mit modernen Gesundheitssystemen verlangen für einen Titel in Hausarztmedizin (HAM) eine strukturierte WB von 5(-6) Jahren Dauer, in der Regel aufgeteilt in 2 Jahre klinische Grund-WB und 3 Jahre spezifische WB [3]. Neben diesen Hausärzten mit einer spezifischen WB sind auch praktische Ärzte (min. 3 Jahren WB) und, v.a. im deutschen Sprachraum, Allgemeininternisten in der ärztlichen Primärversorgung tätig.

\section{Das Weiterbildungsprogramm «Allgemeine Innere Medizin"}

In der Schweiz ist, seit dem Jahre 2011, das WBP AIM für die WB von Hausärzten mit 5-jähriger WB-Dauer zuständig. Die WB gliedert sich in einen Pflichtteil von 3 Jahren Basis-WB in Allgemeiner Innerer Medizin und in 2 Jahre Aufbau-WB nach indvidueller Präferenz. Die Basis-WB beinhaltet 2 Jahre stationäre AIM (davon 1 Jahr in einer A-Klinik) sowie mindestens $1 / 2$ Jahr ambulante WB (in einer Poliklinik oder Praxisassistenz). Eine spezifische WB für die berufliche Laufbahn «Hausarzt» ist jedoch optional. Ein Erwerb von Kenntnissen und Fähigkeiten in der späteren beruflichen Tätigkeit, WB in Chirurgie inklusive Notfall sowie der Erwerb von klinischer Breitenkompetenz durch Kurzrotationen in verschiedenen Fächern werden lediglich empfohlen [4].

\section{Zahlen und Erfahrungen 2011-2016}

\section{Anzahl der neuen Fachärzte AIM}

Die Anzahl der FA AIM [5] insgesamt stieg im ambulanten Sektor von 5800 auf 5921 (+2\%), im stationären von 1603 auf 2295 Ärzte (+43\%, Tab. 1). Im Segment der «unter 65-Jährigen" sank im ambulanten Sektor die Anzahl Ärzte um 336 (-6\%), im stationären stieg sie um 685 (+43\%). Bei den Ärzten im Pensionsalter stieg die Anzahl im ambulanten Sektor um 457 (+44\%), im stationären um 7 (+16\%).

\section{Breitenkompetenz der neuen FA AIM}

Eine Analyse der WB-Stationen der neuen FA AIM zeigt, dass nur jeder zehnte neue FA AIM eine WB gemäss den Empfehlungen absolvierte - in absoluten Zahlen weniger als 30 pro Jahr. Der Anteil der FA AIM mit chirurgischer WB nahm von $55 \%$ auf $39 \%$ ab, der Anteil mit Praxisassistenz verharrte zwischen 30 und 40\% [6].

\section{Unsere Erfahrungen mit der Praxisassistenz}

Die Nachfrage nach Praxisassistenzen schwankte stark. In den Jahren 2010 bis Mitte 2013 hatten wir einen Überhang an Anfragen, von Mitte 2013 bis zu Beginn 2016 meldeten sich kaum Interessenten, seit 


\begin{tabular}{|c|c|c|c|c|c|c|c|c|c|c|c|c|c|c|}
\hline \multicolumn{15}{|c|}{ Fachärzte «Allgemeine Innere Medizin» im Jahre 2011 und 2016 (gemäss FMH) } \\
\hline & & \multicolumn{2}{|c|}{ 5-Jahres-Kohorten } & & & & & & & \multirow[b]{2}{*}{$25-64$} & \multirow[b]{2}{*}{$65-70$} & \multirow[b]{2}{*}{$>70$} & \multirow[b]{2}{*}{ über 65} & \multirow[b]{2}{*}{ Total } \\
\hline & & $25-29$ & 30-34 & $35-39$ & $40-44$ & $45-49$ & $50-54$ & $55-59$ & $60-64$ & & & & & \\
\hline \multirow[t]{2}{*}{ Jahr 2011} & ambulant & & 56 & 395 & 615 & 835 & 966 & 1193 & 1155 & 5215 & 429 & 156 & 585 & 5800 \\
\hline & stationär & 15 & 260 & 461 & 294 & 211 & 145 & 106 & 74 & 1566 & 28 & 9 & 37 & 1603 \\
\hline Jahr 2016 & stationär & 39 & 424 & 647 & 413 & 284 & 213 & 142 & 89 & 2251 & 26 & 18 & 44 & 2295 \\
\hline \multirow[t]{2}{*}{ Delta 2011 / 2016} & ambulant & 1 & 30 & 3 & 33 & -69 & -46 & -219 & -69 & -336 & 281 & 176 & 457 & 121 \\
\hline & stationär & 24 & 164 & 186 & 119 & 73 & 68 & 36 & 15 & 685 & -2 & 9 & 7 & 692 \\
\hline
\end{tabular}

Tabelle 1

Frühling 2016 hält sich bei uns Angebot und Nachfrage etwa die Waage.

Assistenten aus kleineren Spitälern meldeten sich für die Praxisassistenz oft schon in der ersten Hälfte der WB-Zeit und mit klarem Berufsziel «Hausarzt». Sie brachten meist klinische Breitenkompetenz und pragmatisches Arbeiten mit. Assistenten aus grossen Spitälern meldeten sich für eine Praxisassistenz oft am Schluss der WB, bemerkten ihre Defizite (meist fehlende chirurgische WB und klinische Breitenkompetenz) und realisierten, dass es schwierig ist, diese noch wettzumachen. Viele blieben ambivalent in Sachen Laufbahn und wählten als nächsten Schritt eine internistische OA-Stelle oder eine andere ambulante Stelle in einer Spezialdisziplin.

Die Karriere-Aussichten für Ärztinnen mit Kleinkindern, eine wichtige Gruppe der zukünftigen ärztlichen Workforce, war immer schwierig. Sie brachten in der Regel eine gute interdisziplinäre WB mit, der Weg zum FA-Titel AIM bleibt ihnen in der Regel versperrt (zu wenige Teilzeitstellen in A-Klinik).

Auffallend ist das immer noch persistierende inferiore Image der hausärztlichen Laufbahn. Eine junge Kollegin, Fachärztin AIM, brachte es in einem Interview mit Radio SRF [7] gut auf den Punkt:

«Wir [Hausärzte] sollten uns nicht so klein machen. Wir haben eine ganz wichtige Aufgabe gegenüber dem Gesundheitswesen und auch gegenüber unserem Patienten. Und das Problem des Images ist, dass der Hausarzt immer das Gefühl hat: Ja, ich bin ja nur so ein kleiner Hausarzt und die Spezialisten, die sind da eigentlich die richtigen Ärzte. Von diesem Bild müssen wir wegkommen.»

Fazit

Das neue WBP AIM entzieht dem ambulanten Sektor Ärzte und die Breitenkompetenz der WB nimmt ab. Der Erwerb hausärztlicher Kompetenz braucht, im Vergleich zur einer rein allgemeininternistischen WB, einen zusätzlichen Effort - der FA-Titel AIM attestiert allerdings in jedem Falle eine solche Kompetenz, auch wenn sie nicht erworben wurde. Das verunmöglicht nach aussen ein klares Berufsprofil und nach innen eine berufliche Identität.

In diesem WB-Kontext haben die jungen Ärzte wenig Interesse an einer adäquaten hausärztlichen WB und Laufbahn. Das Image ist entsprechend schlecht, die hausärztliche Laufbahn - der «employer of last resort»!

\section{Was ist zu tun?}

Die entscheidenden Faktoren für eine attraktive WB sind: a) einfacher Zugang zu einer adäquaten WB, b) ein klares Berufs-Profil und c) Kennzeichnung der erworbenen Kompetenzen. Zeigt die WB ein klares Profil, wird sich das Image schnell und nachhaltig bessern.

\section{Finalisierung des gemeinsamen WBP}

Ein solches Vorhaben lässt sich am einfachsten mit der Finalisierung des «WBP Allgemeine Innere Medizin» $\mathrm{zu}$ einem, wie ursprünglich anvisiert, gemeinsamen WBP realisieren. Das aktuelle WBP müsste lediglich um ein Kapitel «Pflicht-WB für die hausärztliche Laufbahn» erweitert werden. Gemäss den Empfehlungen (ref 4) könnte eine solche folgendermassen aussehen: a) eine 3-jährige Basis-WB mit 2 Jahren AIM, 6 Monaten interdisziplinärem Notfall (AIM und Chirurgie) sowie 6 Monaten Praxisassistenz und b) eine 2-jährige Aufbau-WB mit neu mindestens 6 Monaten WB in einem weiteren Fach. Alle Ärzte, welche die WB mit der aktuell geltenden Pflicht-WB abschliessen, qualifizieren sich für den «FA Allgemeine Innere Medizin». Diejenigen, welche zusätzlich die Pflicht-WB der «Laufbahn HA» erfüllen, qualifizieren sich für den FA «Allgemeine Innere Medizin und Hausarztmedizin».

\section{Vorteile einer solchen Regelung}

Eine solche Regelung gibt auf individueller Ebene dem «Hausarzt auf FA-Niveau» eine spezifische strukturierte WB, sorgt für ein klares Berufsprofil und damit für eine berufliche Identität, belohnt den zusätzlichen 


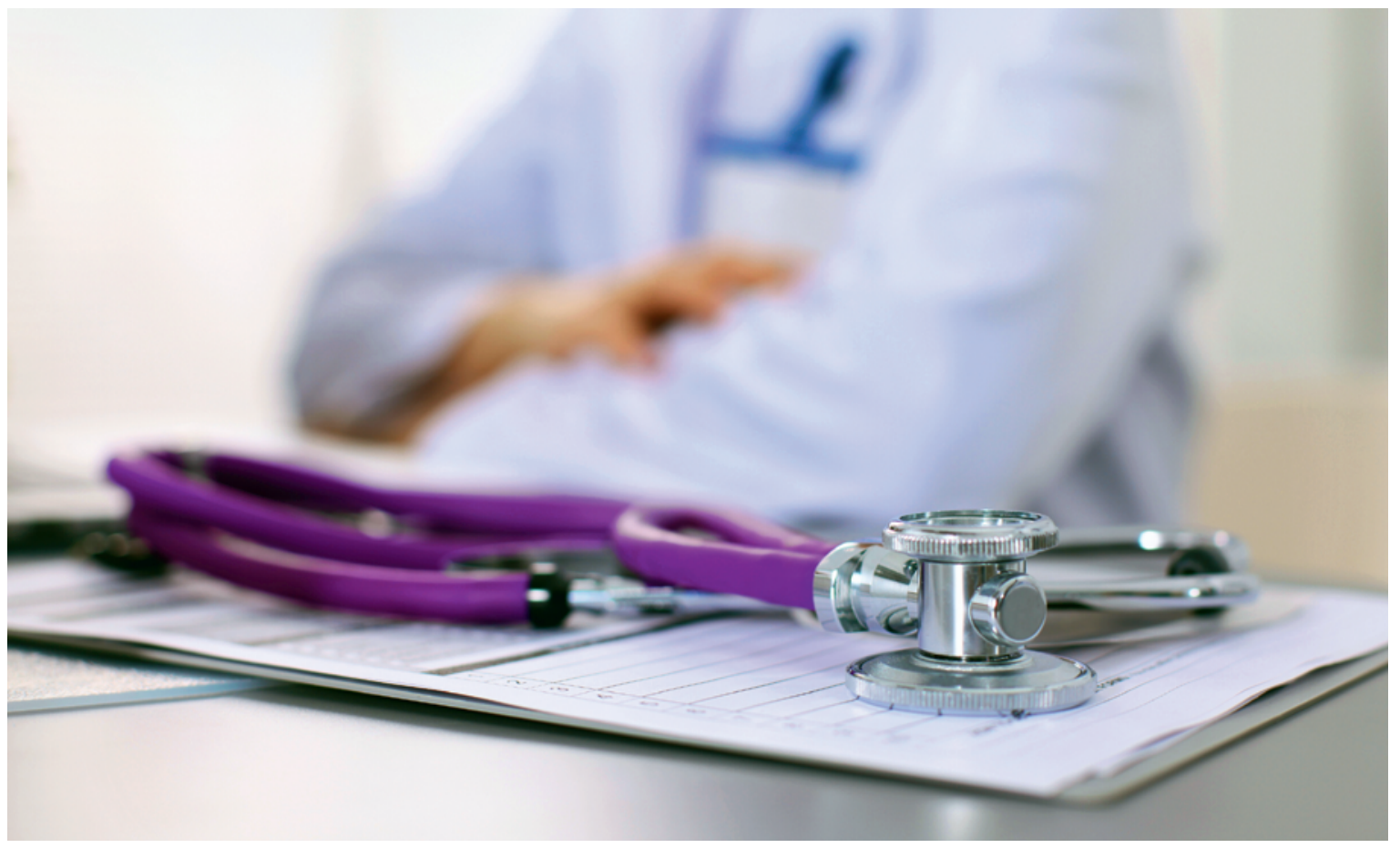

Wie wird die hausärztliche Laufbahn für junge Mediziner wieder interessant?

Effort mit einem definierten Status, macht die fachärztliche Kompetenzen nach aussen sichtbar und erleichtert eine zielführende WB. Auf institutioneller Ebene verzahnt sie den stationären und den ambulanten Sektor besser, z.B. durch Anreize für kombinierte WB-Angebote (z.B. eine A-Klinik bietet A-Jahr mit anschliessender Praxisassistenz). Innerhalb der Medizin fördert sie die Interdisziplinarität und Interprofessionalität und im Rahmen der Gesundheitsversorgung sichert sie den Kompetenz-Erwerb für die hausärztliche Tätigkeit in der Primärversorgung.

Eine solches Vorgehen schafft insbesondere keine neuen Probleme: Die aktuelle WB-Struktur bleibt erhalten, die Übernahme bisheriger WB-Prozesse (Prüfungen, Klassifikation WB-Standorte u.a.) ist möglich und die Kompatibilität mit der EU bleibt gewahrt. Auch die 'Durchlässigkeit' bleibt erhalten: Alle FA AIM können, wie bisher, in der ambulanten Primärversorgung tätig sein und alle «Hausärzte auf Facharzt-Niveau» sind auch FÄ AIM.

\section{Zum Schluss}

Bei der Entwicklung eines gemeinsamen WBP war man 2010 auf halbem Wege stehen geblieben. Das WBP AIM bleibt weit hinter dem Vorschlag der beiden Genfer Allgemeininternisten Humier und Stalder aus den 90er Jahren (Tronc commun) [8] zurück. Es marginalisiert die Disziplin «Hausarztmedizin», zum Nachteil aller: Die AIM beraubt sich damit eines starken Standbeines in der nicht-internistischen Medizin; der ambulanten
Grundversorgung fehlt interdisziplinäre Kompetenz und Erfahrung in interprofessioneller Zusammenarbeit; der Medizin das interdisziplinäre Fach, mit starkem Bezug zu den sozialen Wissenschaften und interprofessioneller Verankerung in der Nahtstelle von Medizin und Gesellschaft.

Eine Weiterentwicklung des aktuellen WBP wäre auf einfache Art möglich und könnte den schon 2010 erhofften Schub für die Hausarztmedizin, aber auch das Allgemeine in der Medizin, stationär und ambulant, doch noch schaffen.

\section{Bildnachweis}

@ Nikolai Lenets | Dreamstime.com

\section{Literatur}

1 Zeller A, Tschudi P. «Anamnese und Status» bei Schweizer Hausärzten. PrimaryCare. 2016;16(15):277-80. https://primary-hospital-care.ch/de/article/doi/phc-d.2016.01327/. 2 Gisler LB, Bachofner M, Moser-Bucher CN, Scherz N, Streit S. From practice employee to (co-)owner: young GPs predict their future careers: a cross-sectional survey. BMC Fam Pract. 2017;18(1):12. doi: 10.1186/s12875-017-0591-7.

3 Djalali S, Fre, A, Rosemann T, Tandjung R. [Vocational training for general practitioners - comparing Switzerland with otherEuropean countries]. Praxis. 2013;102:327-33. doi: 10.1024/1661-8157/a001225.

4 Bürgi U, Tschudi P, Briner V,Héritier F. Das neue Weiterbildungsprogramm Allgemeine Innere Medizin. PrimaryCare. 2010;10: 285-7. https://primary-hospital-care.ch/de/article/doi/pc-d.2010.08696/

5 FMH-Ärztestatistik 2017. Available from: https://www.fmh.ch/ services/statistik/aerztestatistik.html

6 Zahlen von der Befragung durch WB-Kommission SGAM/SGAIM an der FA-Prüfung (nicht publiziert).

7 Hausarzt oder Hausärztin: Beruf oder Berufung? Echo der ZeitRadio SRF, Minute 4:34.

8 Humier JP, Stalder S. Pérspectives pour la formation postgraduée de premier recours. http://www.hausarztmedizin.org/fileserver/ hs.pdf. 